\title{
ВЛИЯНИЕ УПРУГИХ ВОЗМУЩЕНИЙ СТЕНКИ УГЛЕРОДНОЙ НАНОТРУБКИ НА КАНАЛИРОВАНИЕ В НЕЙ МЕДЛЕННЫХ АТОМНЫХ ЧАСТИЦ
}

\author{
А.В. Степанов \\ Кафредра высшей математики и фризики \\ Чебоксарского политехнического института (филиала) МГМУ (МАМИ), \\ ул. П. Лумумбы, 8, Чебоксары, Россия, for.antonstep@gmail.com
}

\begin{abstract}
С помощью классического молекулярно-динамического моделирования исследовано каналирование ионов низких энергий под углами, близкими к критическим, в углеродных нанотрубках. Было обнаружено снижение потерь энергии ионного рассеяния в возмущенных УНТ. Обсуждается механизм каналирования частиц и взаимодействие с возмущениями стенок УНТ: при движении частицы синфазно с волной частица может получать от волны некоторую долю энергии, при этом потери энергии частицей на неупругих столкновениях со стенкой уменьшаются
\end{abstract}

\section{Введение}

Каналирование атомных частиц низких энергий в углеродных нанотрубках (УНТ) изучалось в работах [1-4]. Исследователями [5] было отмечено, что при каналировании частиц под углами, близкими к критическим, в нанотрубке могут возникать солитоноподобные волны упругих возмущений. Солитоноподобные возмущения распространяются вдоль оси УНТ со скоростями, близкими к скорости распространения фононов (10-16 км/с) [6]. В работе [7] было выяснено, что упругие возмущения и ионы $\mathrm{Ar}^{+}$, каналируемые с низкими энергиями (около 100 эВ) могут взаимодействовать между собой. Интерес вызывает режим, в котором каналируемая частица движется синфразно с вызванным ей возмущением стенки УНТ: в этом случае частица теряет меньше энергии на столкновениях со стенкой, чем в случае отсутствия взаимодействия с вызванной ей волной.

\section{Основная часть}

Для выяснение особенностей каналирования атомных частиц в указанном режиме проводились расчеты методом классической молекулярной динамики [8] с использованием потенциала AIREBO [9] для моделирования взаимодействия атомов углеродной нанотрубки. Для взаимодействия атомов углеродной нанотрубки и каналируемой частицы применялся потенциал ZBL [10]. B расчете рассматривались УНТ примерно одинакового диаметра $(10,10),(17,0)$ и $(11,9)$ и равной длины 14.5 нм. Длина выбиралась из условия, что частица должна испытать минимум три столкновения со стенкой и при этом не может быть меньше длины наибольшего из векторов трансляции исследуемых УНТ. Перед расчетом производилась оптимизации геометрии УНТ и минимизация ее энергии методом сопряженных градиентов [11]. Температура трубки поддерживалась на уровне 300 К в течение 5 пс с помощью термостата Берендсена [12], а затем в течение 5 пс с помощью термостата Нозе-Хувера [13]. После релаксации фллуктуации температуры не превышали 5 К. Ионы $\mathrm{Ar}^{+}$запускались с оси УНТ под углами 10-30. Для учета геометрии УНТ азимутальный угол варьировался в диапазоне 0-36 ${ }^{\circ}$, изменялось положение вдоль оси Z так, чтобы покрыть площадь гексагональной ячейки стенки УHT
Анализировались траектории каналированных частиц, при этом по геометрическим и энергетическим характеристикам устанавливался факт столкновение иона $\mathrm{Ar}^{+}$со стенкой. Рассчитывалась полная энергия каналируемого иона после каждого столкновения. На Рис. 1. показана зависимость потерь энергии от угла влета иона в УНТ с усреднением по положению вдоль оси Z и азимутальному углу. Сравнение кривых после первого, второго и третьего столкновения в возмущенной и невозмущенной трубках позволяют судить о влиянии возмущений УНТ на потери энергии частицы. Воздействие возмущений УНТ проявляют зависимость от температуры УНТ, расчеты, проведенные для $0.1 \mathrm{~K}$ почти не дают различий между соударениями и, следовательно, не показывают влияния возмущения стенки УНТ на потери энергии каналируемой частицей. На Рис. 1 отложена кривая потерь энергии иона $\mathrm{Ar}+$ после третьего столкновения с учетом торможения на электронном газе. Видно, что значения потерь энергии близки к аналогичной кривой без учета торможения на электронах УНТ.

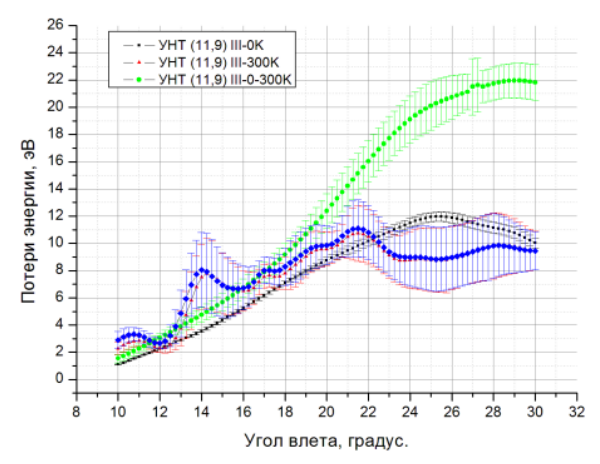

Pис. 1. Потери энергии ионом Ar+ в нанотрубки $(11,9)$ после третьего столкновения со стенкой для УНТ при 0.1 К (квадраты), 300 К (треугольники) и в невозмущенной УНТ при 300 К (круги). Ромбами обозначены потери после третьего столкновения с учетом торможения на электронном газе.

\section{Заключение}

В работе [7] был предложен механизм объяснения взаимодействия частицы и возмущения стенки УНТ, основанный на понятии глиссирова- 
Секция 2. Радиационные эффекты в твердом теле

ния каналируемой частицы на волне упругого возмущения. Так при движении частицы синфазно с волной частица может получать от волны некоторую долю энергии, при этом потери энергии частицей на неупругих столкновениях со стенкой уменьшаются.

\section{Список литературы}

1. Krasheninnikov, A.V. and K. Nordlund. Channeling of heavy ions through multi-walled carbon nanotubes. // Nuclear Instruments and Methods in Physics Research Section B: Beam Interactions with Materials and Atoms, 2005. V. 228. № 1-4. P. 21-25.

2. Krasheninnikov, A.V. and K. Nordlund. Multiwalled carbon nanotubes as apertures and conduits for energetic ions. // Physical Review B. 2005. V. 71. № 24. P. 245408.

3. LI Yong, Z.L.-P., ZHANG Wei**, XU Zi-Jian ${ }^{\star *}$, REN CuiLan, HUAl Ping, ZHU Zhi-Yuan, Charge and Mass Effects on Low Energy lon Channeling in Carbon Nanotubes. // Chin. Phys. Lett. 2011. V. 28. № 6. P. 66101-066101.

4. Zhu, Z., et al. The experimental progress in studying of channeling of charged particles along nanostructure. 2005.

5. Mišković, Z.L. Ion channeling through carbon nanotubes. // Radiation Effects and Defects in Solids 2007. V. 162. № 3-4. P. 185-205.
6. Saito, R., G. Dresselhaus, and M.S. Dresselhaus. Physical properties of carbon nanotubes. 1998, London: Imperial College Press.

7. Степанов A.B. Влияние упругих возмущений стенки углеродной нанотрубки на каналирование в ней медленных атомных частиц. // Поверхность. Рентгеновские, синхротронные и нейтронные исследования. 2015 (в печати).

8. Plimpton S. Fast Parallel Algorithms for Short-Range Molecular Dynamics. Journal of Computational Physics, 1995. V. 117. № 1. P. 1-19.

9. Stuart, S.J., A.B. Tutein, and J.A. Harrison. A reactive potential for hydrocarbons with intermolecular interactions. // The Journal of Chemical Physics. 2000. V. 112. № 14. P. $6472-6486$.

10. Ziegler, J. and J. Biersack, The Stopping and Range of Ions in Matter, in Treatise on Heavy-Ion Science, D.A. Bromley, Editor. 1985, Springer US. P. 93-129.

11. Hestenes, M.R. and E. Stiefel. Methods of conjugate gradients for solving linear systems. I/ Journal of Research of the National Bureau of Standards. 1952. V. 49. № 6. P. 409-438.

12. Berendsen, H.J.C., et al. Molecular dynamics with coupling to an external bath. // The Journal of Chemical Physics. 1984. V. 81. № 8. P. 3684-3690.

13. Hoover W.G. Generalization of Nosé's isothermal molecular dynamics: Non-Hamiltonian dynamics for the canonical ensemble. // Physical Review A. 1989. V. 40. № 5. P. 2814-2815.

\title{
INFLUENCE OF CARBON NANOTUBE'S ELASTIC PERTURBATION ON SLOW ATOMIC PARTICLES CHANNELING
}

\author{
A.V. Stepanov \\ Department of Mathematics and Physics, Cheboksary Polytechnic Institute (branch) MGMU \\ (MAMI), Str. Lumumba, 8, Cheboksary, Russia, for.antonstep@gmail.com
}

Low energy ion channeling with angles close to critical in carbon nanotube with help of classical molecular dynamic simulation was investigated. Lower energy losses ion scattering with perturbed CNT wall was detected. We discuss mechanism of channeling particle and CNT wall perturbation interaction. This mechanism is close to hydroplaning effect. When channeling particle slide down the CNT wall perturbation wave. If particle moves in phase with perturbation wave particles energy losses decrease compared to not perturbed CNT. 